\title{
Los juegos florales y la creación del valor literario. El caso de la narrativa breve antioqueña ${ }^{1}$
}

\author{
Gustavo Adolfo Bedoya Sánchez ${ }^{2}$
}

Resumen. Este artículo describe y analiza las formas en que la institución literaria ha creado y dotado de valor a los autores y a su producción. Utiliza como ejemplo el caso de la narrativa breve antioqueña, bajo la celebración de los juegos florales; y se basa en la sociología de la literatura, la cual concibe la producción del valor literario como parte de los mecanismos de consagración.

Palabras clave: literatura colombiana; narrativa breve; juegos florales; sociología de la literatura; valor literario; consagración.

\section{[en] Floral games and the creation of literary value. The case of the Antioquia brief narrative}

\begin{abstract}
This article describes and analyzes the ways in which the literary institution has created and endowed with value to authors and their production. It uses as an example the case of the Antioquia brief narrative, under the celebration of the floral games; and is based on the sociology of literature, which conceives the production of literary value as part of the mechanisms of consecration.

Keywords: Colombia Literature; Brief Narrative; Floral Games; Sociology of Literature; Literary Value; Consecration.
\end{abstract}

Sumario. 1. Introducción 2. Los juegos florales 3. La invitación 4. La celebración 5. Institucionalización y valoración literaria 6. Palabras finales.

Cómo citar: Bedoya Sánchez, G.A. (2018) Los juegos florales y la creación del valor literario. El caso de la narrativa breve antioqueña, en Anales de Literatura Hispanoamericana 47, 53-72.

Despierto está el entusiasmo por tan civilizadores torneos en los principales centros de Colombia. Fue Medellín el primero que los celebró en el pasado mes de Octubre, con inusitada pompa; con no menor entusiasmo lucieron después en Bogotá, y ahora se preparan Manizales y Sonsón á celebrarlos. (Lectura y Arte, 1904: 143-144). ${ }^{3}$

1 Este artículo se deriva de la investigación "El cuento colombiano en las revistas literarias colombianas (19001950). Estudio histórico y hemerográfico”, se inscribe, además, en el marco de la Estrategia de Sostenibilidad para grupos de investigación CODI 2016-2017 (Universidad de Antioquia).

2 Universidad de Antioquia, Medellín. Colombia.

E-mail: gustavo.bedoya@udea.edu.co

3 Hemos conservado la ortografía y la redacción original de las fuentes históricas consultadas. 
Débesele [al Centro Artístico] la implementación en Medellín de los tradicionales Juegos Florales, bellísima ocasión que motiva el estímulo y el entusiasmo para la carrera de las

Letras, y hace surgir hombres y nombres nuevos, de indiscutible notoriedad, á la vez que proporciona al público veladas dignas de capitales cultas. (Lectura y Arte, 1906b: 221).

\section{Introducción}

Pensada como una práctica social, la literatura es un proceso colectivo. En su concepción intervienen diferentes agentes e instancias, por ejemplo: los críticos y editores, así como los distintos modos de publicación y difusión (Dubois [1979] 2014: 86-93). De esta manera, la literatura no es el resultado -único- de un escritor; ella es la definición que las instituciones le confieren y resuelven usar. La literatura también es una práctica histórica, es decir, cambiante. En ella interfieren las condiciones temporales y espaciales en las que se desarrolla. Como nunca es la misma, tampoco lo es la intervención que en ella hace la "institución literaria”.

Dado lo anterior, y para la sociología de la literatura, la institución es quien “elabora”, “define” y “legitima” la producción (Dubois [1979] 2014: 70-85). Desde este punto de vista, el "valor" de lo literario no puede darse por sentado, ni depende únicamente de los procesos de creación. Para Pierre Bourdieu, por ejemplo, el "valor" de la literatura es un resultado de los mecanismos propios del "campo literario”; así las cosas, es tarea del estudioso analizar la ‘invención’ del valor literario, sus formas y mecanismos:

Partiendo de que la obra de arte sólo existe como objeto simbólico provisto de valor si es conocida y está reconocida, es decir si está socialmente instituida como obra de arte por unos espectadores dotados de la disposición y de la competencia estéticas necesarias para conocerla y reconocerla como tal, la ciencia de las obras tendrá como objeto no sólo la producción material de la obra sino también la producción del valor de la obra o, lo que viene a ser lo mismo, de la creencia en el valor de la obra ([1992] 1995: 339).

Así, estas páginas se proponen describir y analizar las formas en que la institución literaria ha creado y dotado de valor a los productores y a la producción literaria. Utiliza como ejemplo el caso de la narrativa breve producida en la región antioqueña, bajo la celebración de los juegos florales. En Colombia, estas fiestas fueron ideadas en el siglo XIX, pero sólo se concretaron a principios del siglo XX.

Ya que en nuestro contexto no existen investigaciones sistémicas sobre los concursos literarios, ni tampoco existen archivos dedicados al tema de los juegos florales, hemos optado por recurrir a la prensa, testigo discursivo de los acontecimientos históricos. Como órgano de difusión de las sociedades literarias del pasado, la prensa es vista como protagonista del devenir histórico de lo literario; en esta medida, la descripción y el análisis que hacemos de los juegos 
florales recorre, cronológicamente, el camino trazado por las publicaciones periódicas literarias de Antioquia. ${ }^{4}$

\section{Los juegos florales}

La historia de los juegos florales es de antigua data, tanto que podríamos denominarla una historia de longue durée, en términos de Fernand Braudel ([1949] 1987: 17). Algunos estudiosos relacionan estas celebraciones con el culto al dios Dionisio, en Grecia; y con el culto a la diosa Flora, en Roma, donde se conocieron como Ludi Floreales (Scullard 1981: 110). Otros llaman la atención sobre las fiestas dedicadas a la gaya ciencia ("alegre ciencia”, tal como era conocida la poesía), las cuales tuvieron lugar durante la Edad Media, en Francia, y que parecen ser el antecedente más importante de los actuales juegos. ${ }^{5}$

Para nuestra investigación, los juegos florales representan, además de una fiesta y un concurso, una de las tantas estrategias que la institución literaria tiene para "presentar" y "promocionar" a los creadores y a sus obras (Dubois [1979] 2014: 73 y 82). No debe olvidarse que la celebración de los juegos hace que la sociedad centre su atención en el fenómeno literario y en todo lo que la literatura significa, o debe significar para sus agentes. Estas celebraciones mantienen viva la tradición literaria y actualizan el protagonismo que la institución cree que el escritor y su obra se merecen. En términos conceptuales, todo concurso literario debe ser pensado como una estrategia de “consagración”. ${ }^{6}$

\section{La invitación}

En Colombia, y hasta donde sabemos, la primera mención a los juegos florales se hizo el 18 de julio de 1868, en El Oasis. Periódico Literario, de Medellín. Esta publicación semanal, de ocho páginas, pequeñas y atiborradas de muestras

4 Sobre la importancia de la prensa literaria, y su valor como protagonista histórico, véase las investigaciones conceptuales de Osuna (1998 y 2004). La creación del valor literario tampoco ha sido abordada en nuestro contexto; son los estudios franceses los más representativos, especialmente los que tienen que ver con la invención del valor en los concursos del siglo XX (Ducas 2010, Dozo 2010); para un acercamiento al caso latinoamericano, véase la presentación que hace Fernández (2010).

5 Aunque en las fiestas dionisiacas se celebraron competencias teatrales (cuyo ganador recibía una corona de yedra), fue durante los festejos de Toulouse (1324) que los juegos se concretaron a manera de torneo literario. Éstos encontraron asidero en España: Cataluña (1334), Barcelona (1830), Valencia (1878), Cádiz (1912), Sevilla (1916), así como otros centros urbanos. En América Latina fueron celebrados -por primera vez- en: La Habana (1846), Buenos Aires (1881), Concepción (1884), Quetzaltenango (1916), Lima (1919), Valparaíso (1910), Santiago de Chile (1914), Ciudad de Panamá (1916), Cuzco (1940), Sahuayo (1955), Nueva Friburgo (1960), Querétaro (1965), etc. Por su parte, en Colombia tuvieron lugar en Medellín (1904), Sonsón (1905), Bogotá (1908), Quibdó (1908), Manizales (1910), Aguadas (1911) y Jericó (1914), según nuestras pesquisas iniciales.

6 Por ejemplo, en 1914 y en 1921, el primer puesto de los juegos florales -organizados por la Federación de Estudiantes de la Universidad de Chile-, le fue otorgado a Gabriela Mistral y Pablo Neruda, respectivamente. Ella tenía 25 años y él 17. Ambos recibieron la Flor Natural y contaron con la oportunidad de ocupar un lugar entre los poetas nacionales y sus obras se sumaron a la producción literaria chilena. No sobra decir que ambos autores, posteriormente, fueron galardonados con el Premio Nobel de Literatura, ella en 1945 y él en 1971, pero sus presentaciones públicas se hicieron en las justas nacionales. 
literarias, venía acompañada de un generoso 'editorial', el cual -en ciertos momentos- llegó a ocupar más de una cuartilla. Fue en una de estas notas donde apareció la mención exacta a los juegos florales; allí se daba noticia histórica de la primera celebración, acaecida en Europa, en 1324.

Es necesario aclarar que la nota publicada en El Oasis reproduce información tomada de un periódico español, cuyo título no se detalla; asimismo, la nota establece que los primeros juegos tuvieron lugar en Tolosa, España; cuando realmente sucedieron en Toulouse, Francia. Otro tanto hace cuando declara que la señora Clemencia Isáura instauró la celebración para eternizar su memoria; realmente fue Clemence Isaure, quien instituyó los juegos florales donando su dinero y casa para que las celebraciones continuaran. ${ }^{7}$ El Oasis relacionó las características que definieron la inauguración de los primeros juegos, características que siguen siendo las mismas al día de hoy. ${ }^{8}$ Finalmente, El Oasis termina admirando la ejecución de los juegos "españoles”, al grado de atreverse a lanzar una propuesta, pensando siempre en la "ilustración” del pueblo antioqueño:

Juzgamos que no será difícil establecer en Antioquia una asamblea literaria compuesta de todas las personas que se han dedicado al cultivo de las bellas letras, señalando un día para reunirse todas en esta ciudad en el lugar previamente convenido. Una vez reunidos los que quisiesen hacer parte de esta asamblea, podría dársele formas organizándola de la manera más conveniente; i verificando esto cada miembro de ella sabría lo que debía hacer en lo futuro ( $E l$ Oasis, 1868b: 226).

Para nuestra investigación es importante reconocer, junto con María Cristina Arango, que El Oasis fue "El primer periódico de literatura editado en Medellín” (Arango 2006: 62). Bajo la coordinación de Isidoro Isaza -quien suponemos fue el autor de los ‘editoriales'-, el periódico se ocupó de ofrecerle un espacio a la literatura regional y a la exposición y evaluación de las costumbres sociales. Así, literatura y buenas costumbres parecen ser los ejes centrales de esta publicación. En sus páginas se publicaron narrativas breves y una cantidad ingente de poemas, marcados por el respeto moral: "Queremos decir que El Oasis será pura y exclusivamente literario, procurando que su estilo sea tan decente que la virgen

Como no se indica el título de la publicación española, no hemos logrado constatar la información para saber a quién pertenecen las inexactitudes; ahora bien, en Revista de Ciencias, Literatura y Artes, de Sevilla (España), aparece una carta de José Fernández-Espino, en la que se da noticia de los juegos llevados a cabo en Córdoba (España), en 1859. En esta carta se cometen las mismas irregularidades (Fernández-Espino 1859: 493).

8 Por lo regular, para la ejecución de los juegos florales se confía en un "comité" organizador, dividido según sus funciones. Casi siempre se cuenta con un "presidente” y un "secretario". Además, el comité selecciona a los “jurados”, quienes se encargan de evaluar las obras participantes. La celebración tiene lugar en un sitio reconocido, y durante los días de fiesta se organizan diversas actividades que acompañan y complementan la celebración: el discurso inaugural, el informe de los jurados, la lectura de las obras ganadoras, la elección de la "reina"; y también: eucaristías, cenas, espectáculos musicales y artísticos, etc. Los reconocimientos incluyen, las más de las veces, una estatuilla -símbolo de alguna flor-, nombrada además con la mención a un metal precioso, por ejemplo: "la violeta de oro”. En los juegos se busca la premiación por una obra literaria, regularmente un poema o un cuento, pero existen casos en los que se premian las obras teatrales, novelísticas y ensayísticas. A estas participaciones se les ha exigido ser inéditas, estar escritas correctamente y, especialmente, no atentar contra la moral y las buenas costumbres. 
más pudorosa pueda leerlo sin experimentar el más leve sonrojo” (El Oasis, 1868a: 1).

Por su parte, para Luis Latorre Mendoza, en las páginas de El Oasis se dieron cita "todos los literatos de la época, buenos, regulares y malos" (Latorre [1934] 1972: 243). Según nuestras indagaciones, el periódico publicó textos de, entre otros: Ricardo Campuzano, Juan José Botero, Manuel Uribe Ángel, Cipriano Mejía, Gregorio Gutiérrez González, Demetrio Viana, Pedro A. Isaza, Domingo D. Granados, Epifanio Mejía, Camilo Antonio Echeverri, Juan C. Arbeláez, Basilio Tirado, Ricardo López, Federico Jaramillo, Pedro Bravo, Federico Velásquez y Vicente A. Montoya. Podemos decir que estos nombres representan parcialmente-, el origen de la literatura antioqueña. Dada la época, ninguno de ellos logró dedicarse por entero a lo literario, pero en conjunto encarnan los albores de la institución literaria.

Según Dora Helena Tamayo y Hernán Botero Restrepo, la joven literatura antioqueña se "origina y configura” entre 1833 -fecha de aparición de $L a$ Miscelánea de Antioquia-, y los años finales del siglo XIX (2005: xiii). En la falta de experticia que caracterizó a este momento inaugural tiene lugar el proyecto periodístico de Isidoro Isaza; de allí que El Oasis resulte un medio periódico formativo: permite la escritura de los novatos, al tiempo que educa a su público lector. Estos dos propósitos, bien pueden verse traducidos -también- en el proyecto de celebración de los juegos florales. ${ }^{9}$

Una vez hecha la invitación a organizar los juegos, El Oasis sugirió celebrarlos el 20 de julio, durante las festividades propias de la Independencia. La idea era conjugar patriotismo y literatura. El periódico también llamó la atención sobre la necesidad de organizar un "consejo" encargado de la propia ejecución de las celebraciones (El Oasis, 1868b: 226).

Durante los siguientes dos meses, y sin mencionar explícitamente los juegos, El Oasis se dedicó a demostrar que Antioquia ya tenía todo lo necesario para llevarlos a cabo: argumentó sobre la importancia de utilizar la "narrativa" para celebrar la Independencia (1868c: 233-234), le dedicó un 'editorial' a los jóvenes comprometidos con el desarrollo de la sociedad (1868e: 257), enfatizó en el rol de la mujer como fuente de inspiración estética (1868d: 229-230) y productora de literatura (1868i: 305), así como resaltó el "talento" (1868f: 265-266), la “conducta” (1968g: 273) y la "voluntad” (1868h: 281-282) de los jóvenes antioqueños. ${ }^{10}$

Ahora bien, la invitación de El Oasis no logró ningún resultado. Nueves meses después reimprimió su invitación, recordando la facilidad de organizar los juegos.

9 Para comprender el estado inicial de la literatura antioqueña es necesario hacer alusión a sus limitadas condiciones: en términos materiales, las editoriales e imprentas eran pocas, así como el tiraje de los medios periódicos; además, la escritura no era considerada una profesión y la lectura era un privilegio de pocos, en una sociedad mayoritariamente analfabeta e interesada en el enriquecimiento material.

10 La formación literaria de la mujer, ejercida por El Oasis, es evidente en el listado de suscriptoras, compuesto mayoritariamente por las "señoras” y "señoritas” de Antioquia; asimismo, El Oasis indicó publicar los ejercicios literarios de algunas mujeres, no conocidas en el ámbito literario (1868i: 305); y finalmente, una vez concluido El Oasis, la publicación saludó y presentó La Aurora (Medellín: 1868-1869), del ya nombrado Venancio A. Calle, periódico literario “dedicado al bello sexo” de Antioquia. Por su parte, la nueva publicación hizo el siguiente saludo: "Nosotros queremos coadyuvar la tarea de EL OASIS, y con tal fin fundamos este periódico que será puramente literario” (La Aurora, 1868: 2). 
Para esta ocasión se atrevió a señalar los nombres de quienes podían fungir como jurados: "Residen en la capital del Estado hombres de cuya competencia en asuntos literarios, nadie puede dudar [...] muchos otros, son bien conocidos en Antioquia i fuera de ella, por sus productos literarios" (1869a: 122). ${ }^{11}$ De igual forma, El Oasis se atrevió a solicitar la ayuda expresa de la sociedad medellinense y estableció una cita, el 27 de mayo, para que los colaboradores del periódico pudieran votar públicamente en la elección de los cinco jurados (también podían votar por correspondencia). El ‘editorial' recordó que una vez escogidos los jurados, debía pensarse en el nombre de los juegos y en los premios. De inmediato, declara que los juegos pueden contar con las páginas del periódico para hacer pública la obra ganadora, como recompensa. Otra vez llama la atención sobre el ejemplo “español”, e insta a que los medellinenses dejen la "pereza” y participen: "Ningun sacrificio hay qué hacer para lograr lo que nos proponemos: unos pocos instantes robados a nuestras ocupaciones o a nuestros pasatiempos, serán mas que suficientes para ello" (1869a: 122).

Resulta indiciario que durante la reunión citada no se haya logrado concretar ninguna propuesta; sin embargo, los asistentes consiguieron reflexionar sobre el "retraimiento" de los medellinenses, y aunque no desecharon la idea de los juegos, plantearon modificarla dados los recursos "intelectuales i pecuniarios con que contamos", así, consideraron necesario organizar -primero-, una junta "preparatoria" (El Oasis, 1869b: 169).

A pesar de los dos llamados, y la constante defensa por la celebración de los juegos, El Oasis recibió su única respuesta en mayo, diez meses después de hacer la invitación. La respuesta tiene forma de misiva, escrita por "Orión” (seudónimo de Juan José Molina), a su amigo "Eduardo" (quizás, Eduardo Villa). La carta dicta que la invitación de El Oasis, de la cual habla "toda la ciudad", no dista mucho de su idea de fundar una sociedad literaria. Tal sociedad podría hacerse cargo de celebrar los juegos cada año, dice Orión, aunque sabe que dicho intento puede resultar infructuoso (tal como ya había sucedido en el pasado, al intentar organizarse). Ahora bien, Orión sabe que en esta ocasión la ciudad cuenta con "dos periódicos" literarios, lo que facilitaría potencialmente la publicación de las muestras. El remitente enfatiza en que la literatura no es una "profesión" en Antioquia y que no lo será hasta dentro de mucho tiempo: la literatura antioqueña está en la "infancia, i no serán inútiles los esfuerzos que se hagan por crearla" (Orión 1869: 163. El énfasis es nuestro).

Tenemos que decir que la carta de Orión no tuvo respuesta pública, sin embargo, El Oasis se encargó de dar noticia de todo lo referente a la organización de la sociedad. Así, su 'editorial' del 26 de junio toca dos temas: el primero está relacionado con el "sueño" de fundar un Instituto de Bellas Artes; el segundo, informa acerca de las reuniones para organizar la sociedad: "Ojalá que todas las personas que tienen derecho a concurrir, lo hagan con constancia para que no fracase por decidia (sic), tan bella como útil empresa" (El Oasis, 1869c: 202). Fundar un instituto, concretar los juegos florales y organizar a los agentes literarios

11 Se trata de, los ya citados, Gregorio Gutiérrez González, Camilo Antonio Echeverri, Manuel Uribe Ángel, Demetrio Viana, Epifanio Mejía, Juan José Molina, Pedro A. Isaza, así como de Cipriano Mejía y Antonio María Restrepo Restrepo (más conocido como Abel Farina). 
son muestra clara de la consciencia que la joven literatura antioqueña tenía sobre la necesidad de su organización, de su institucionalización.

Dos meses después, en agosto, El Oasis notificó la instalación de El Casino de la juventud, fundado por Federico Velásquez y otras "personas notables". Informa que a la inauguración concurrieron más de 50 personas, "jóvenes en su mayor parte", y que el discurso inaugural estuvo a cargo de Demetrio Viana (1869d: 265). En septiembre, la tertulia tenía 93 asistentes, todos caballeros "escrupulosamente escojidos" (1869e: 500).

A pesar del número de participantes, El Casino de la juventud tampoco logró celebrar los juegos, así como El Oasis y La Aurora no lograron sobrevivir y tuvieron que cerrar su edición, ante los típicos problemas económicos (representados en el bajo número de suscriptores y el no pago de los abonos). Otro tanto puede decirse del sueño frustrado de fundar un Instituto de Bellas Artes. Dada esta realidad, podemos decir que el inicio de la historia de los juegos florales es la historia de los intentos por organizar a la ciudadanía, exactamente a los escasos hombres de letras de la ciudad, quienes surgen apáticos e invadidos por la desidia. También es la historia de los intentos de formación, tanto de un público lector, como de los propios escritores. Por su parte, la literatura de las buenas costumbres (civilista y patriótica), apta para las mujeres y “la virgen más pudorosa”, deberá encontrar otros medios periódicos para su publicación, otros ‘oasis literarios’. La oferta no es amplia, pero mejorará con los años. En principio la prensa sólo le ofrecerá a la literatura un espacio reducido, pero para finales del siglo XIX encontremos medios dedicados por entero a ella ${ }^{12}$.

\section{La celebración}

Los primeros juegos florales de Colombia tuvieron lugar en Medellín, en el mes de octubre de 1904 (36 años después de la invitación de El Oasis). Entre uno y otro momento podemos registrar significativos cambios, materiales e intelectuales, pero todos ellos se pueden resumir en la fundación de la Sociedad de Mejoras Públicas. Esta institución permitió que lo literario alcanzara un protagonismo que nunca había tenido. Ella fue una iniciativa de Carlos E. Restrepo, futuro presidente de Colombia (1910-1914), quien junto a un poco más de veinte socios, en febrero de 1899, concretaron una sociedad por fuera del Estado y la Iglesia. Su objetivo estaba cifrado en la modernización y ornato de la ciudad, así como en la modernización del pensamiento. Dado lo último, idearon el Centro Artístico, fundado en 1904 y

12 Durante la segunda mitad del siglo XIX, en Medellín, tuvieron lugar diversas empresas periodísticas donde lo literario jugó un papel importante, a saber: El Cóndor (1870-1871), de Juan C. Aguilar; y El Álbum (18721873), de Demetrio Viana, Clodomiro Castilla, Juan José Molina y Francisco de Paula Muñoz. También vale la pena mencionar los periódicos escolares: La Palestra (1872), de Silvestre Balcázar; La Lechuza (1875), de un grupo de estudiantes de la Universidad de Antioquia; y El Liceo Antioqueño (1884), de Juan José Molina, órgano del Liceo de Antioquia. Son de mayor importancia los medios culturales: Novedades (1877-1910), de Nazario A. Pineda; y especialmente: La Miscelánea (1886-1901, 1903-1914), de Juan José Molina, en la que se estrenaron escritores de la talla de Efe Gómez, Tomás Carrasquilla y Francisco de Paula Rendón; sus páginas se nutrieron de las discusiones que tenían origen en la Librería Restrepo, de Carlos E. Restrepo. Otro tanto debe decirse de las revistas ilustradas: El Repertorio (1896-1897), de Luis de Greiff; y El Montañés (1897-1899), de Gabriel Latorre, Francisco Gómez y Mariano Ospina. 
dirigido por el reconocido empresario Ricardo Olano (quien además coordinó su medio periódico, la revista Progreso). ${ }^{13}$

El Centro Artístico contó con la participación de, entre otros: Luis de Greiff, Rafael Montoya Pérez, Jorge de la Cruz, Julio Vives Guerra, Carlos Melguizo y Antonio José Montoya. Entre muchas otras actividades, logró ejecutar los juegos florales; fundó el, antiguamente soñado, Instituto de Bellas Artes; y apoyó la publicación de Lectura y Arte, la primera revista de arte publicada en Medellín.

Lectura y Arte, tal como lo hacía la prensa cultural de la época, declaró no pertenecer a ningún partido político. En su primer número suplicó la "benevolencia" ante lo "modesto" de su proyecto, y estableció las normas de escritura: no ofender la moral y escribir "corto", "claro" y "culto" (Lectura y Arte, 1903: 3). Publicó noticias y textos sobre arte, además de dibujos y pinturas; trató temas coyunturales, como las negociaciones del canal de Panamá y la desidia cultural de los medellinenses; y editó muestras poéticas y narrativas de autores nacionales y extranjeros. ${ }^{14}$ Un hecho a resaltar: antes de llevar a cabo los juegos florales promovió otros concursos para ilustrar sus portadas; asimismo, la publicación logró una división interna -en sus funciones-, mucho más profesional que los medios periódicos decimonónicos: Francisco A. Cano y sus discípulos, Marco Tobón Mejía y Enrique Vidal, se hicieron cargo de lo relacionado con el arte; el poeta y librero Antonio J. Cano coordinó el aspecto literario; y en Francisco A. Latorre recayeron las actividades administrativas.

Como medio periódico del Centro Artístico, Lectura y Arte tomó atenta nota de todo lo relacionado con los juegos florales. Así las cosas, sabemos que el acuerdo fundacional de los juegos fue firmado por Ricardo Olano y Benjamín Tejada Córdoba, en sus respectivas funciones de presidente y secretario. En este documento se establecieron las características generales de la celebración, a saber: el Centro Artístico es la institución que organizará los juegos, anualmente, en el mes de mayo. Para ello, en el mes de enero, se publicarán las condiciones de participación. Los tres jurados principales, y los tres suplentes, deberán pertenecer al Centro Artístico y serán elegidos en el mes de marzo. Asimismo, serán ellos quienes deberán escoger cuáles obras pueden ser leídas durante los días de las “fiesta”. En el acuerdo también se establecen los premios: una "violeta de oro" y "diploma de honor", al que componga la mejor obra en prosa o verso; una "violeta de plata”, para el segundo puesto; y un "diploma de honor”, para el tercer puesto. También se establece que los jurados pueden hacer mención de las obras que consideren. Por otro lado, el autor galardonado será el encargado de elegir a la "Reina de los Juegos”, quien a su vez elegirá a su "corte”. Los autores que logren ganar tres veces quedarán por fuera del concurso, para permitir la participación de otros autores. En relación con las obras, el acuerdo establece que la "temática" es "libre”, al igual que la "forma": "El solo criterio admisible al juzgar de ellas, será

13 Los proyectos de la Sociedad lograron la transformación de la ciudad y su gente. Existe un estudio enfocado en ella (García, 1997) y nosotros hemos tenido la oportunidad de establecer su papel en la concreción de medios periódicos y en la fundación de comunidades intelectuales (Bedoya 2015a y 2015b).

14 En narrativa sobresalen: Saturnino Restrepo, Gregorio Martínez Sierra, Alfonso Castro, Antonio José Montoya, Samuel Velásquez, Clímaco Soto Borda, Rafael Montoya Pérez; y Guy de Maupassant, Vicente Blasco Ibáñez y Mark Twain. En poesía: Abel Farina, Víctor M. Londoño, Adolfo León Gómez, Max Grillo, Guillermo Valencia, Santiago Pérez Triana, Julio Vives, Isaías Gamboa, Aquilino Villegas, Alfredo Gómez Jaime; y Gabriel D’Annnunzio, Paul Bourget y Alfred Tennyson. 
el de que sean obras de ingenio y de arte, originales é inéditas” (Olano y Tejada 1905: 158).

Los primeros juegos florales fueron inaugurados con las palabras de Luis de Greiff, quien saludó al "selecto” y "numeroso" público; luego aludió al papel protagónico del Centro Artístico (concretado gracias a "unos pocos” que aún defienden el arte). De Greiff explica que los juegos florales son otro intento más por incentivar la creación literaria antioqueña; así que llama la atención sobre las "glorias literarias" existentes (a saber, los copartícipes de El Oasis): Gregorio Gutiérrez González, Epifanio Mejía y Camilo Antonio Echeverri, además de Emiro Kastos y Juan de Dios Uribe. Dados estos nombres, el orador se permite una apreciación sobre la calidad estética de las obras presentadas: "Empero, no debéis exigir obras maestras á una sociedad incipiente como la nuestra, y al dar vuestro juicio, debéis hacerlo con criterio amplio y sereno y sin olvidar el nivel relativamente bajo que marca el cultivo de las letras en nuestro suelo" (De Greiff 1905: 160). Para explicar las lamentables condiciones artísticas del departamento, el autor alude a las consecuencias de la guerra, el marasmo intelectual, la ambición de lucro y el derrumbamiento de todo lo noble, como lo son las "fruiciones inefables del arte”. Sus palabras finalizan anudando la educación estética junto con la educación moral, en un principio cristiano: amar a la humanidad y no solamente la vida propia. ${ }^{15}$

Por su parte, el jurado calificador de los primeros juegos estuvo conformaron por Gabriel Latorre, Carlos A. Molina, Francisco Cano y Luis de Greiff (en reemplazo de Carlos E. Restrepo). Los jurados empiezan disculpándose por no ser “expertos” en el tema literario (esta aclaración se convertirá en una constante en las demás justas). En su informe exponen que recibieron 50 participaciones: 24 en verso, 25 en prosa, y un "cuaderno" provisto de ambas formas textuales. Cada jurado, en privado, leyó la totalidad de las muestras; y luego, en grupo, confrontaron sus opiniones. Al final se establecen a los ganadores (es necesario apuntar que la revista publicó las obras ganadoras, pero no las honoríficas). En narrativa, el primer puesto fue para Juan de Dios Vásquez, con su obra "Arroyo" (Vásquez 1905: 166-168); el segundo fue para Rafael Montoya, por "Martita" (Montoya 1905: 171-175). La primera obra es calificada de "pulcra", dueña de un estilo exacto en el uso de las palabras: "no escribe para niños de escuela y se hace leer entre líneas” (Latorre y otros 1905: 163). El segundo cuento es calificado de "sueño amoroso", con cierto estilo "picaresco". Los jurados, además, deciden otorgar dos menciones honoríficas, a dos cuentos firmados con seudónimos. Sus autores no aclararon sus nombres, así que se desconocen sus autorías. ${ }^{16}$

Ya que se trata de una celebración que consagra, es de suma importancia señalar que Lectura y Arte -además de publicar las obras premiadas-, publicó los retratos

15 Aunque las condiciones sociales de este momento son distintas a las registradas a mediados del siglo XIX, la ciudad vive la recuperación de diversas guerras civiles, así como no logra compensarse ante los altos niveles de pobreza y analfabetismo.

16 Por su parte, en poesía el primer puesto fue para Eusebio Robledo, con “El Palacio del Arte” (Robledo 1905: 166-168); el segundo para Antonio J. Cano, con "Crespúsculos” (Cano 1905: 170). Se hizo mención honorífica a: “¡Es un rayo de luna!”, de Julio Vives Guerra; y “Obsesión”, firmado por “Moi”, e identificado con el nombre Moi-meme ("Yo mismo”, en francés), de allí que también se desconozca su verdadera autoría. 
de los ganadores (así como los retratos de la Reina y su corte). ${ }^{17}$ Ahora bien, en los juegos sucedió algo mucho más relevante para nuestra investigación: entre las obras presentadas un cuento sobresalió por encima de los cuentos ganadores, y aunque fue publicado junto con el retrato de su autor, no recibió distinción alguna. Estamos hablando de "Post Mortem”, de Enrique Gaviria (Gaviria 1905: 168169).$^{18}$ En su informe, los jurados manifiestan que la obra posee un alto valor literario, pero dada su temática -y el giro que la historia toma-, no resulta adecuado para ser leído públicamente. Los jurados le dedican más atención a este cuento que al resto de las obras. Lo juzgan de estudio psicológico breve, pero de concentrada energía; anotan que es un episodio humano en toda su amplitud; y que la narración revela un conocimiento acertado del corazón humano; así como evidencia las dotes de observación de su autor y su desempeño en el uso de la "palabra"; cierran asegurando la "concomitancia" que el relato guarda entre "prosa” y "asunto":

Por su pensamiento y por su forma, consideramos, pues, de mérito sobresaliente esta hermosa producción. Desgraciadamente, la pieza aunque moral en el fondo, tiene una conclusión que -dada la susceptibilidad y delicadeza de nuestras costumbres- tal vez pudiera ser en pública lectura causa de rubores, y es por esto por lo que el Jurados, atendiendo á la $4^{\mathrm{a}}$ de las condiciones del Concurso, que exige que las composiciones sean de tal naturaleza que puedan leerse ante un público compuesto de personas de ambos sexos, se ve obligado, muy á su pesar, á desecharla, aunque no sin proclamar, como expresamente lo hace, la superioridad de sus méritos” (Latorre y otros 1905: 162-163).

Tal como lo expondremos más adelante, las decisiones del jurado calificador suscitaron algunas críticas; las cuales hoy consideramos reacciones certeras que garantizan a nuestro entender el éxito logrado en las justas. Ahora bien, a pesar de los logros obtenidos, el Centro Artístico no pudo concretar la celebración de los segundos juegos en 1905, y apenas en febrero de 1906 da noticia de ellos. Lectura y Arte invita a la participación exponiendo los nombres de los nuevos jurados, a saber: Fidel Cano, Carlos E. Restrepo y Félix Betancourt; y como suplentes: Sebastián Hoyos, Luis de Greiff y José Montoya (1906a: 214). Es necesario anotar que, para este mismo momento, Lectura y Arte anuncia su cierre, no sin antes relacionar las actividades ejecutadas por el Centro Artístico: exposiciones y conferencias públicas, además de los juegos florales. Se nos dice que la institución sigue a cargo de Ricardo Olano, en compañía de Luis de Greiff, de quienes se incluyen retratos (1906b: 221).

Lectura y Arte se despidió calificando su labor de "improductiva” (1906c: 214). Debemos decir que ella logró editarse durante un poco más de dos años y medio, pero sólo publicó doce números recurriendo, en tres ocasiones, a la edición de números dobles. En términos generales, y tal como lo hemos querido exponer en

17 Véase los retratos de los ganadores, en: Lectura y Arte, nº. 9-10, abril, pp. 161, 164, 170 y 171; y los retratos de las "Reinas": la "Señora, Doctora, Clara Jaramillo de Robledo”; “las señoritas, doctoras, María Mendoza y Alicia Mendoza”; y la “señorita, doctora, Magdalena Montoya”, en Lectura y Arte, nº. 9-10, abril, pp. 159, 166, 167 y 174, respectivamente.

18 Véase el retrato del autor, en: Lectura y Arte, nº. 9-10, abril, pp. 178. 
estas páginas, Lectura y Arte -gracias al apoyo del Centro Artístico-, logró reunir y dar cuenta de una sociedad literaria mucho más moderna que los diversos intentos decimonónicos. Existe una gran diferencia entre ella y sus antecesoras, no sólo por ejecutar y dar cuenta de los juegos florales, sino también por el espacio que le ofreció a los autores nacionales e internacionales, y no sólo antioqueños. Otro tanto puede decirse por su énfasis artístico y porque sus textos críticos tienden a ser más actuales (en cuanto a su evaluación) y menos formativos.

En relación con los juegos florales, su ejecución es ya el mayor de los logros, así como la publicación de las obras ganadoras. Otro hecho importante radica en que, en esta ocasión, la celebración de los juegos no se relacionó con ningún asunto civil y mucho menos patriótico. La organización y diferenciación de funciones, en el interior de la celebración, también es un síntoma del profesionalismo con que fueron asumidos; así como todo lo relacionado con la propia fiesta: la evaluación, los premios, el público y la elección de las reinas, etc.

Por último, es evidente la diferencia que existe entre la institución literaria formada por El Oasis, frente a la institución que posibilitó el Centro Artístico, de la Sociedad de Mejoras Públicas. Aunque en ambos momentos tengamos que seguir hablando de una literatura joven, para principios del siglo XX el sistema literario empezó a coleccionar autores de sumo valor, como lo son Saturnino Restrepo y Abel Farina, además del reconocido Tomás Carrasquilla. Ahora bien, hay una característica que sigue uniendo a estas dos literaturas, característica que fue evidente en la celebración de los juegos: ambas literaturas siguen siendo moralistas, en términos temáticos, y ambas aspiran a hacer un uso correcto, gramaticalmente, del lenguaje. Estos dos momentos, como lo hemos visto, hacen alusión a la imagen de la virgen que se "sonroja" y a los "rubores" que la lectura puede desencadenar entre el público femenino, respectivamente. De este hecho es sintomática la decisión de los jurados, quienes no premian un cuento por su "asunto", aunque resulte una muestra ejemplar de calidad literaria en su "concomitancia", tal como ellos mismos lo dicen, entre "prosa" y "asunto"; o en términos teóricos bajtinianos del siglo XX, entre forma y contenido (Bajtín [1924] 1975: 13-75).

\section{Institucionalización y valoración literaria}

El ya nombrado Centro Artístico concretó sus esfuerzos, en pro de lo literario, en la publicación de la revista literaria antioqueña más importante: Alpha (Medellín: 1906-1912, 1915). En ella se asumió lo literario, y su evaluación, desde un sentido mucho más moderno. A diferencia de sus antecesoras antioqueñas, Alpha abrió sus páginas a muestras literarias internacionales (lo que le permitió una conciencia sobre la importancia de la traducción); así como a un sentido profesional de la crítica literaria, en la que se sucederán discusiones de autores tan importantes para el género crítico, como las mantenidas por Tomás Carrasquilla, Max Grillo, Baldomero Sanín Cano y Saturnino Restrepo. ${ }^{19}$ La revista también permitió la

19 En otro momento tuvimos la oportunidad de estudiar la crítica literaria en la prensa colombiana de principios del siglo XX (Bedoya y Barrios 2015). 
discusión sobre problemas sociales que afectaban, de manera directa, la educación y el arte.

Alpha fue anunciada en el último número de Lectura y Arte, y en aras de llamar la atención sobre su potencial valor, se establecieron los nombres de sus coordinadores, los ya nombrados: Antonio J. Cano y Luis de Greiff, además de Ricardo Olano, quien con toda su experticia asumió la "gerencia" del proyecto (Lectura y Arte, 1906c: 214). Para la Medellín de principios del siglo XX, estos nombres tenían un valor simbólico que -de antemano-, hizo pensar a muchos en el éxito de la empresa. Por su parte, y en su primer número, Alpha invitó a los colaboradores de Lectura y Arte a pertenecer al nuevo proyecto (Alpha, 1906a: 44); otro tanto hizo con los colaboradores de la revista literaria de Colombia más importante para el momento: Revista Contemporánea (Bogotá: 1904-1905), del crítico literario nacional por antonomasia, el antioqueño Baldomero Sanín Cano.

Es necesario anotar que Alpha declaró no tener intenciones "docentes", y que sólo quería ser un esfuerzo contra los "inapetentes intelectuales" de la sociedad (Alpha, 1906b: 41). Igual que su antecesora, Alpha informó sobre los proyectos del Centro Artístico -entre ellos-, los relacionados con los juegos florales.

La celebración de los segundos juegos tuvo lugar en mayo de 1906. Para esta ocasión Luis Eduardo Villegas fue invitado a pronunciar el discurso inaugural, en el que empieza disculpándose por su falta de autoridad. Seguidamente reconoce la “inteligencia” y “comprensión” de los jurados: Fidel Cano, Sebastián Hoyos (quien reemplazó a Carlos E. Restrepo) y Félix Betancourt. Por último, establece las características de debe tener una obra literaria: moral intachable y uso adecuado de los preceptos gramaticales y retóricos (Villegas 1906: 236). Esta visión resulta idéntica a la establecida en los primeros juegos y la expuesta en la prensa literaria; sin embargo, Villegas agrega: una obra literaria debe contener "sentimiento" y "fantasía": "que son los grandes manantiales de la estética". Lo anterior lo obliga a precisar: la obra debe "pintar" la "naturaleza tropical propia”, sin "embellecer" la realidad; ejemplifica aludiendo a la obra de Dante, Cervantes y Goethe, quienes produjeron impresiones de fealdad, despertando al mismo tiempo emociones artísticas. Por otro lado, sigue puntualizando: la obra literaria no debe caer en la pintura de lo "exótico", ni tampoco debe ser tan "enigmática" que "ni el propio autor" la pueda entender. De esta forma, concluye: una obra literaria debe ser "verdadera”, "artística” y “clara”.

Los jurados del encuentro también se disculpan por la "inmerecida distinción" que les hacen al convocarlos como evaluadores. Agradecen a Ricardo Olano y al Centro Artístico todos los esfuerzos por llevar a cabo las justas. Aluden a la actual situación de la sociedad que rechaza el arte en favor de lo práctico: "En el lenguaje de quienes dirigen la cruzada antiliteraria, escritor es casi término injurioso, poeta voz despectiva, y uno y otro vocablo suenan á holgazanería y á carencia de verdadero patriotismo" (Cano y otros 1906: 177. El énfasis en el original). Luego aluden al proceso de evaluación (idéntico al del primer concurso) y establecen los ganadores. Aquí es necesario puntualizar que los jurados decidieron no otorgar ningún premio en poesía, sólo menciones honoríficas, dado el poco valor de las propuestas; en contraposición, los jurados tuvieron problemas a la hora de descartar los cuentos que merecían ganar: tanto en número como en calidad, las obras en prosa "resultaron ganadoras" (179-180). Aquí es necesario decir que, para este 
momento histórico, en Lectura y Arte y Alpha, no se distingue una clara diferencia conceptual entre "prosa", "cuento" y "narrativa", términos que la mayor de las veces son utilizados como sinónimos. No sucede lo mismo con otros géneros, pues es fácil advertir la diferencia que hacen entre "cuento" y "poesía", o más específicamente, entre "cuento" y "novela”, ya que sólo la primera es "narrativa”, en alusión a una muestra breve, escrita en prosa

No está de más decir que Alpha se encargó de publicar gran parte de las obras seleccionadas. En prosa publicó el primer puesto y la mención honorífica, a saber: "Baldosas y terrones", de Jorge de la Cruz (De la Cruz 1906: 186-204); y "La oveja descarriada”, de Saturnino Restrepo (Restrepo 1906: 206-231), respectivamente. Además, los jurados "enaltecieron" nueve obras, dos de las cuales publicaron: "Martín Rúa”, de Gaspar Chaverra (Chaverra 1906: 257-266); y “Nobleza obliga”, de J. A. Gaviria (Gaviria 1906: 334-356). Las otras siete obras fueron mencionadas sin indicar su autor, éstas son: "Paréntesis moral", "Manolo", "Sepulcros blanqueados", "Sor Angélica”, “Arpa ingente”, "Realismos” y "Risas". ${ }^{20}$

Para esta segunda celebración no se incluyeron los retratos de los autores galardonados, ni tampoco información distinta a la evaluación y premiación. Y ya que no se entregó la "Flor natural" -el primer puesto en poesía-, no hubo ocasión para escoger a la Reina. Los jurados conocían el riesgo de hacerlo, ya que estas decisiones podían quitarle "atractivo" al acto festivo; sin embargo -según sus palabras-, las decisiones fueron tomadas pensando en la literatura, y no en la fiesta (Cano y otros 1906: 180-181). Los jurados dicen estar convencidos de que en Antioquia se pueden producir mejores obras poéticas, que las presentadas, y esperan que para otros certámenes los participantes se esmeren.

Los jurados también se permitieron reflexionar sobre el mayor uso de la prosa, frente a la poesía. Aseguran que, dada la juventud literaria de los autores, éstos prefieren las formas "libres", “amplias” y "robustas”, de "fácil energía”, como las de la prosa y la narrativa; en contraposición a las formas "suaves" y "delicadas" de la poesía. Los jóvenes -dicen los jurados-, prefieren esquivar "las dificultades que halla el pensamiento para acomodarse en los estrechos, precisos y afiligranados moldes métricos” (Cano y otros 1906: 182). También aseguran que la sociedad antioqueña ofrece más material para llevar a la prosa, y menos para la poesía, de allí que los poetas que "quedan" deban cantar a lo exótico: al mar, a la estepa y al desierto (sin conocerlos), mientras los prosistas pueden cantarle al "terruño", "y de allí su fuerza, vigor y energía crecientes” (Cano y otros 1906: 184). Aquí podemos advertir una idea generalizada sobre ambas formas literarias, ideas que harán academia, aún hoy en día: con regularidad se piensa que la poesía es una forma de mayor cuidado y destinada para los expertos, mientras la prosa hace parte, apenas, de los iniciales ejercicios de escritura.

Hay una gran diferencia entre la primera y la segunda celebración de los juegos florales. En este momento, los jurados del Centro Artístico tienen una conciencia mayor sobre el aspecto literario, y ya no tanto sobre la celebración y la fiesta. Así, estos juegos están más cerca del torneo y del concurso, que de la festividad. Esta

20 En poesía los jurados llamaron la atención sobre siete obras, todas ellas con mención honorífica. Alpha publicó dos de ellas: el poema de Antonio J. Cano, “Sendero de llanto” (Cano 1906: 205); y la obra de Efraím de la Cruz, “Anima” (De la Cruz, Efraím, 1906: 232-233). Los otros poemas mencionados, sin los nombres de sus autores, son: “Himno vernal”, "Flor de ensueño”, “Margarita”, “Memento” y “Una vieja hoja de misal”. 
dinámica intentará ser conservada en los siguientes juegos. Lo interesante aquí es que, para este momento, el Centro Artístico ya ha institucionalizado los juegos florales como algo propio, como parte de sus funciones anuales. No sólo los ejecutó en Medellín, sino también en Jericó, una ciudad secundaria del departamento (en donde, inicialmente, se enfocaron en la celebración, más que en el torneo). De allí que el nombre de los juegos florales parezca unido al nombre del Centro Artístico. Luego de la segunda fiesta, el Centro Artístico los celebró en seis ocasiones más, en Medellín; y en tres ocasiones en la ciudad de Jericó. ${ }^{21}$

Por otro lado, las decisiones de los jurados son altamente significativas para nuestro estudio porque son sus críticas las que crean las obras. En términos teóricos: para que un discurso exista requiere un "metadiscurso" que le otorgue reconocimiento (Dubois [1979] 2014: 80). De esta forma, los jurados de los juegos florales están en la misma posición que los críticos y editores. Estos agentes tienen, o deberían tener, una definición de lo literario que les permite codificar y reproducir las normas que rigen la producción. En sus prácticas como jueces, aseguran la circulación y el uso de las obras. De esta forma, su función principal es la legitimidad y la consagración; así como los concursos, premios y festividades literarias, y en este caso los juegos florales, hacen parte de las estrategias de presentación y promoción, tanto del autor como de su obra. Otro tanto puede decirse de la revista literaria como microsistema, en la que internamente se reconoce al autor y a la obra; de la misma forma en que las celebraciones y las publicaciones periódicas funcionan como lugares de encuentro e intercambio literario.

Así las cosas, llama nuestra atención los momentos en que jueces y críticos reconocen a los escritores de los juegos florales. Según Luis de Greiff, el crítico Baldomero Sanín Cano reconoció el valor literario de Juan de Dios Vásquez, situándolo “á la cabeza de los cuentistas antioqueños” (De Greiff 1905: 161). Otro tanto hace José Montoya, en su “Crónica literaria” de Alpha, en la que expone:

Ayer no más empezamos esta implantación [de los juegos florales], y yá nos dio el fruto de conocer y estimular al joven escritor Rafael Montoya Pérez, cuyo nombre van pregonando por doquiera sus propias obras, no las voces adulonas de amigos que mutuamente se elogiaron como fue usanza cuando faltaba el mérito. ¡Quién sabe qué nueva sorpresa nos guarda el certamen próximo! [...] (Montoya 1906: 40).

Otro tanto sucedió durante la tercera celebración de los juegos florales, evento que contó con el discurso de apertura de Félix Betancourt, quien además de agradecer la designación reconoce su falta de experticia. Su discurso versó sobre las condiciones que hacen de Antioquia una tierra próspera para el progreso. El autor llama la atención sobre la geografía particular del departamento, así como apunta algunas consideraciones acerca de la "raza", pero en seguida advierte los

21 Los ganadores del primer puesto en prosa, en Medellín, fueron: Alfonso J. Gómez (1912), Romualdo Gallego (1917 y 1925) y Libardo Parra Toro (1918). No conocemos los ganadores de los juegos celebrados en 1933 y 1950, aunque sí sabemos que el crítico Rafael Maya fue invitado en 1933. Por su parte, en Jericó se celebraron en 1914, 1975 y 1998. Toda la información sobre los eventos en esta ciudad se encuentra en Jericó, medio periódico del Centro de Historia de Jericó. 
ejemplos de quienes han hecho del departamento un lugar ejemplar, tanto en la pintura, como en la música y la literatura. Así, en el caso literario señala los nombres de personalidades tales como Gregorio Gutiérrez González y Epifanio Mejía, pero también los nombres de Tomás Carrasquilla, Abel Farina y Efe Gómez; en cierto sentido, los nombres más reconocidos de la literatura antioqueña de finales del siglo XIX y principios del XX. Entre todos ellos, también señala a los autores que representan, en su actualidad, una joven tradición de renombre, dada la calidad de sus obras. Entre estos autores menciona a algunos colaboradores del Centro Artístico: Gabriel Latorre y Samuel Velásquez, por ejemplo; pero más importante, menciona a los autores galardonados en los juegos florales, a saber: Antonio J. Cano, Julio Vives Guerra, Gaspar Chaverra y Jorge de la Cruz; y también a Alfonso Javier Gómez y Alfonso Castro, quienes con sus obras "Madre glotona” y "Abismos sociales", respectivamente, obtuvieron el primer y segundo puesto en la categoría narrativa, de los juego florales de 1912 (Betancourt 1912: 126).

Justamente, sobre los dos últimos autores arriba señalados, Tomás Márquez compuso una serie de apuntes críticos. Acerca de la obra de Alfonso Javier Gómez apunta que adolece de un uso exagerado del adjetivo. El crítico argumenta que la forma no es tan importante como el contenido, para ello utiliza el paralelo entre Flaubert y Maupassant. Aconseja al joven escritor estudiar la vida con ojo perspicaz, en lugar de preocuparse por los "dandysmos del lenguaje”, de esta manera logrará escribir con "vigor y soltura” (Márquez 1912: 156). Por su parte, califica de "preciosa" la obra de Alfonso Castro, y se dedica a desatender la crítica que -dice- realizó Baldomero Sanín Cano sobre ella. Para Sanín Cano la obra es "local" y sus personajes no se corresponden con las cosas "elevadas" que pronuncian; en cambio, para Márquez el arte no puede ser "fotográfico", y la obra de Castro logra retratar las miserias de la vida social, así como logra ser una crítica a la educación moral y material de la mujer. En el culmen de su argumentación, Márquez compara la obra de Castro con "Grandeza”, de Tomás Carrasquilla. Finalmente, Márquez alude a la naturaleza del ejercicio crítico, dice que ha evaluado según sus "ideas estéticas” y su honradez, y que Nietzsche se equivoca al hablar de la alegría de la crítica, ya que ella es -también- un terreno difícil, igual que la creación.

Por su parte, José Montoya, en su “Crónica literaria”, publicada en Alpha, se dedica a evaluar la obra de Alfonso Castro, llamando la atención sobre su importancia para la vida literaria antioqueña. Expone la grandeza que representa "Hija espiritual", a la cual pone por encima de Kundry, la novela de Gabriel Latorre (Montoya 1906: 30-40). Ya en otro momento el crítico había establecido que los "cuentos modernos" de Castro, publicados en Notas humanas, resultaban mucho más importantes que la colección de cuentos El Recluta, en la que $\mathrm{La}$ Miscelánea publicó a los reconocidos Samuel Velásquez, José Antonio Gaviria y Tomás Carrasquilla (1903: 17). 


\section{Palabras finales}

Para nosotros, la historia de los juegos florales es -también- la historia de la literatura, de sus posibilidades y limitaciones. Una historia literaria que, además de enfocarse en los autores y las obras, lo hace en los críticos, editores y jurados como instancias de reconocimiento y consagración. De igual forma, los juegos florales representan la historia de los imaginarios y las sensibilidades en torno al fenómeno estético. Si bien es cierto que los juegos estimulan a sus autores con el reconocimiento, ellos también representan un espacio de sociabilidad en donde los autores novatos se encuentran con los afamados $y$, todos ellos, con el público que aplaude admirado.

En términos ideológicos, los juegos permiten promover, defender, enseñar y difundir una lengua y su uso estético; así como hace otro tanto con costumbres, valores e, incluso, ideales compartidos. También celebran el papel inspirador de la mujer, la belleza y el amor; y especialmente: los juegos permiten el desarrollo de la creación literaria, ellos ponen en primer plano todo lo relacionado con lo literario.

En nuestra historia de los juegos florales hemos señalado tres momentos específicos: un llamado a la ejecución de los juegos, la celebración misma y el instante en que estos juegos se convirtieron -conceptualmente-, en un campo posibilitador de la consagración literaria. En estos tres momentos el fenómeno literario fue facilitado por la acción conjunta de los agentes literarios, quienes tuvieron que lidiar de maneras distintas frente a las condiciones sociales y a las necesidades específicas del campo literario.

Para la sociedad literaria antioqueña los juegos se convirtieron en una necesidad: a mediados del siglo XIX, dada la realidad inicial de la literatura, los juegos iban a permitir la concreción del fenómeno literario, tal como lo estaba intentando hacer -con sus propios medios-, la prensa literaria. El énfasis estuvo cifrado en la formación de los agentes y en la urgencia de reconocerse, ellos mismos, como los protagonistas del hecho literario. La institución literaria tuvo dificultades para ser ella misma, para inventarse. Ella encontró un espacio de acción mínimo, circunscrito a las publicaciones periódicas, pero no por fuera de ellas. El proyecto de Isidoro Isaza, El Oasis, representa justamente eso, un oasis en una ciudad analfabeta y apática frente al arte, interesada en el enriquecimiento material y no 'espiritual'.

Durante la celebración de los primeros juegos florales, la institución literaria encontró reposo en la fundación del Centro Artístico. Una vez inventada, y una vez sus copartícipes se reconocieron entre ellos mismos, el Centro Artístico ejecutó los juegos florales y posibilitó -además-, el Instituto de Bellas Artes y la publicación de diversos medios periódicos, entre los que hemos señalado Lectura y Arte y Alpha. Ahora bien, fue necesario un cambio en la perspectiva para que los juegos florales dejaran de enfocarse en las celebraciones per se, para centrarse en el concurso y en la evaluación del talento.

Las reseñas, notas y textos críticos, así como los informes de los jurados, marcan la existencia de las obras literarias al nombrarlas (incluso, tan sólo al mencionarlas, positiva o negativamente). Es en este momento en el que tiene lugar el nacimiento del autor y su obra. 
Resulta imperativo mencionar que, en el mismo momento en que la crítica crea al autor y a la obra, el crítico mismo se está reforzando en su propia existencia. Su texto es prueba patente que demuestra su categoría de evaluador. Otro tanto sucede cuando un segundo agente llama la atención sobre los juicios de un primer crítico. En este sentido, resulta interesante evidenciar la manera en que la sociedad literaria antioqueña, de principios del siglo XX, se auto-referencia en la sucesión de obras literarias y anotaciones críticas. De esta manera, en la concreción del valor literario no sólo es suficiente anotar las obras; también se las compara con textos de mayor autoridad, por ejemplo, con Tomás Carrasquilla; y se alude a los comentarios que han hecho otros críticos, entre ellos el crítico de mayor prestigio: Baldomero Sanín Cano. Estas acciones son las que permiten establecer, justamente, el valor de las obras.

Hay un hecho que nos permitiremos resaltar porque, hoy en día, no ha sido estudiado en nuestra academia, nos referimos a las “dedicatorias” expresas que los autores deciden hacer en sus obras. Resulta de supremo valor comprobar la manera en que las dedicatorias dibujan una red de agentes en la que es posible evidenciar a los protagonistas de la institución literaria. En esta práctica un autor reconoce a otro, ya sea su valor literario, su autoridad en algún tema, o incluso su amistad y su cariño. Una mención somera de las dedicatorias impresas en las obras ganadoras de los segundos juegos florales nos permite llamar la atención sobre el grado de cofradía entre los escritores galardonados y el propio Centro Artístico. La institución literaria crea el funcionamiento literario de sus agentes y hace posible que, entre ellos mismos, se reconozcan y se consagren gracias a los agradecimientos y a las menciones. Por ejemplo: Saturnino Restrepo le dedica su obra a Ricardo Olano, Gaspar Chaverra a Pablo Echevarría, Antonio J. Cano a Francisco A. Cano y Efraím de la Cruz a Luis Cano.

Ahora bien, en los términos actuales del valor literario, debemos decir que la literatura producida a principios del siglo XX fue una literatura que se corresponde con las limitaciones ideológicas de la época. La literatura esperada y resultante de los juegos florales es una literatura conservadora que funciona dentro de los parámetros de lo correcto, en términos temáticos-morales y gramaticales. Cualquier trasgresión fue desautorizada, tal como pudimos evidenciarlo. Esta literatura tradicional está muy relacionada con la literatura realista y el cuadro de costumbre, mucho más que con los ejercicios modernistas o experimentales, de allí la sobre atención en los contenidos y no en las formas. Otro tanto debe decirse en la apreciación que se hace sobre el uso de la narrativa frente a la poesía exótica y enigmática, es decir, de tinte modernista.

También es necesario decir que los juegos florales, de principios del siglo XX, permitieron un reconocimiento circunstancial de los autores galardonados y sus obras; pero más importante, permitieron que el Centro Artístico y sus copartícipes adquirieran el reconocimiento y la consagración de 'gestores' culturales, posibilitadores de manifestaciones artísticas y críticos autorizados del conocimiento literario. Los autores que en alguna ocasión recibieron mención en los juegos son recordados, en la actualidad, como gestores y críticos, más que como autores de ficción, el caso de Antonio J. Cano y Saturnino Restrepo, hombres de prensa, cuyos nombres están unidos a los de figuras tales como Carlos E. Restrepo, cabeza del Centro Artístico, que curiosamente no logró ser parte de las 
celebraciones (dadas sus ocupaciones políticas), y al lado de Ricardo Olano, un reconocido industrial que no despreció el arte, y que -por el contrario-, supo 'administrar' las empresas culturales a su cargo, actividad que permitió justamente-, la 'relativa autonomía' de la institución literaria, en la Medellín de principios del siglo XX.

\section{Referencias bibliográficas}

\section{Fuentes primarias}

Alpha, "Para empezar”, Alpha. Medellín, Año I, nº 1, marzo, 1906a, pp. 41-42

- “Homenaje”, Alpha. Medellín, Año I, no. 1, marzo, 1906b, pp. 43-44.

—_Centro Artístico”, Alpha. Medellín, Año I, nº. 4, junio, 1906c, pp. 164.

Betancourt, Félix, "De Antioquia...”, Alpha. Medellín. Año VII, nº. 75-76, agosto, 1912, pp. 106-128.

Cano, Antonio J., “Crepúsculos”, Lectura y Arte. Medellín, nº. 9-10, abril, 1905, pp. 170.

—_Sendero de llanto”, Alpha. Medellín, Año I, no. 5-6, julio, 1906, pp. 205.

Cano, Fidel y otros, “Concursos de 1906. Informe del Jurado Calificador”, Alpha. Medellín, Año I, nº. 5-6, julio, 1906, pp. 177-185.

Chaverra, Gaspar, "Martín Rúa”, Alpha. Medellín, Año I, no . 7, agosto, 1906, pp. 257-266

De la Cruz, Efraím, “Anima”, Alpha. Medellín, Año I, nº. 5-6, julio, 1906, pp. 232-233.

De la Cruz, Jorge, "Baldosas y terrones”, Alpha. Medellín, Año I, no . 5-6, julio, 1906, pp. 186-204.

De Greiff, Luis, "Discurso del Sr. L. de Greiff”, Lectura y Arte. Medellín, nº. 9-10, abril, 1905, pp. 159-161.

El Oasis, “El Oasis”, en El Oasis. Periódico Literario. Medellín, nº 1, 11 de enero, 1868a, pp. 1.

—_El Oasis”, El Oasis. Periódico Literario. Medellín, no. 29, 18 de julio, 1868b, pp. 225226.

—_El Oasis”, El Oasis. Periódico Literario. Medellín, nº. 30, 25 de julio, 1868c, pp. 233234.

—_El Oasis”, El Oasis. Periódico Literario. Medellín, nº. 32, 8 de agosto, 1868d, pp. 229230.

—_El Oasis”, El Oasis. Periódico Literario. Medellín, no . 33, 15 de agosto, 1868e, pp. 257.

—_El Oasis”, El Oasis. Periódico Literario. Medellín, no . 34, 22 de agosto, 1868f, pp. 265266.

—_El Oasis”, El Oasis. Periódico Literario. Medellín, no. 35, 29 de agosto, 1868g, pp. 273.

—_La Voluntad”, El Oasis. Periódico Literario. Medellín, nº. 36, 5 de septiembre, 1868h, pp. 281-282.

—_Escritoras de la Nueva Granada”, El Oasis. Periódico Literario. Medellín, nº. 39, 26 de septiembre, 1868i, pp. 305.

—_El Oasis”, El Oasis. Periódico Literario. Medellín, no. 16, 17 de abril, 1869a, pp. 121122.

—_El Oasis”, El Oasis. Periódico Literario. Medellín, no. 22, 29 de mayo, 1869b, pp. 169170.

— “La educación de los jóvenes”, El Oasis. Periódico Literario. Medellín, nº 26, 26 de junio, 1869c, pp. 201-202.

—“El Casino de la Juventud”, El Oasis. Periódico Literario. Medellín, nº. 34, 21 de agosto, 1869d, pp. 265-266. 
—_El casino de la juventud”, El Oasis. Periódico Literario. Medellín, nº 38, 18 de septiembre, 1869e, pp. 500-501.

Fernández-Espino, José, “Sr. D. Juan Justiniano y Arribas”, Revista de Ciencias, Literatura y Artes. Sevilla. Tomo Quinto, 1859, pp. 491-496.

Gaviria Isaza, Enrique, "Post Mortem”, Lectura y Arte. Medellín, nº 9-10, abril, 1905, pp. 168-169.

Gaviria, J.A., “Nobleza obliga”, Alpha. Medellín, Año I, nº. 8-9, septiembre, 1906, pp. 334356.

La Aurora, "La Aurora. Al bello sexo antioqueño”, La Aurora. Medellín, nº 1, 24 de octubre, 1868, pp. 1-2

Latorre, Gabriel y otros, “Juegos florales. Informe del Jurado calificador”, Lectura y Arte. Medellín, nº. 9-10, abril, 1905, pp. 161-163

Lectura y Arte, "Lectura y Arte”, Lectura y Arte. Medellín, nº 1, julio, 1903, pp. 3

—_Los juegos florales”, Lectura y Arte. Medellín, no. 7-8, noviembre, 1904, pp. 143-144.

— “Jurado”, Lectura y Arte. Medellín, no. 12, febrero, 1906a, pp. 214.

—“Centro Artístico”, Lectura y Arte. Medellín, no. 12, febrero, 1906b, pp. 221.

- “Alpha”, Lectura y Arte. Medellín, nº 12, febrero, 1906c, pp. 214.

Márquez, Tomás, “Dos novelas”, Alpha. Medellín. Año VII, nº 75-76, agosto, 1912, pp. 151-157.

Montoya, José, “Crónica literaria”, Lectura y Arte. Medellín, n. 1, julio, 1903, pp. 16-17 y 19.

—“Crónica literaria”, Alpha. Medellín, Año I, n. 1, marzo, 1906, pp. 30-40.

Montoya Pérez, Rafael, “Martita”, Lectura y Arte. Medellín, n. 9-10, abril, 1905, pp. 171175.

Olano, Ricardo y Benjamín Tejada Córdoba, “Acuerdo Jugos Florales”, Lectura y Arte. Medellín, nº. 9-10, abril, 1905, pp. 158.

Orión, “Carta a un amigo”, El Oasis. Periódico Literario. Medellín, nº. 21, 21 de mayo, 1869, pp. 162-163.

Restrepo, Saturnino, “La oveja descarriada”, Alpha. Medellín, Año I, nº 5-6, julio, 1906, pp. 206-231.

Robledo, Alfonso, “Discurso”, La Miscelánea. Medellín, Año X, nº 1, 1910, pp. 3-5

Robledo, Eusebio, “El Palacio del Arte”, Lectura y Arte. Medellín, no. 9-10, abril, 1905, pp. 164-165.

Vásquez, Juan de Dios, “Arroyo”, Lectura y Arte. Medellín, n. 9-10, abril, 1905, pp. 166168.

Villegas, Luis Eduardo, “Carta”, Alpha. Medellín, Año I, nº. 5-6, julio, 1906, pp. 234-237.

\section{Obras citadas}

Arango, María Cristina. Publicaciones periódicas en Antioquia 1814-1960. Del chibalete a la rotativa. Medellín: Fondo Editorial Universidad EAFIT, 2006.

Bajtín, Mijaíl, "El problema del contenido, el material y la forma en la creación literaria”, en Teoría y estética de la novela. Trabajos de investigación. Madrid: Taurus, [1924] 1975, pp. 13-75.

Bedoya Sánchez, Gustavo Adolfo, “«Biografía» de la revista literaria Alpha (Medellín: 1906-1912, 1915). Formación de una empresa cultural y una escuela discursiva”, en Adriana Pineda Soto (coord.). Recorridos de la prensa moderna a la prensa actual. Morelia (México): Universidad Autónoma de Querétaro, Universidad Michoacana de San Nicolás de Hidalgo, 2015a, pp. 253-276.

— “Entre la teoría y la práctica. La educación en la revista literaria Alpha (Medellín: 19061912, 1915): formación de una comunidad intelectual”, en Arte a seis voces. Colección 
Becas a la creación. Ensayos de crítica en artes. Medellín: Pulso \& Letras Editores, Alcaldía de Medellín, 2015c, pp. 115-145.

Bedoya Sánchez, Gustavo Adolfo y Diana María Barrios, "Entre la norma y la ruptura, entre lo clásico y lo moderno. La crítica literaria colombiana en la prensa de 1900 a 1920”, en Olga Vallejo (ed.). “La busca de la verdad más que la verdad misma”. Discusiones literarias en las publicaciones periódicas colombianas 1835-1950. Lima: Centro de Estudios Literarios Antonio Cornejo Polar, 2015, pp. 121-157.

Bourdieu, Pierre. Las reglas del arte. Génesis y estructura del campo literario. Barcelona: Anagrama, [1992] 1995.

Braudel, Fernand. El Mediterráneo y el mundo mediterráneo en la época de Felipe II. México: Fondo de Cultura Económica, [1949] 1987. Tomo I.

Dozo, Björn-Olav y Michel Lacroix, "Petits dîners entre amis (et rivaux) : prix, réseaux et stratégies de consacrants dans le champ littéraire français contemporain”, COnTEXTES, $\mathrm{n}^{\circ}$. 7, junio (2010). Disponible en: http://contextes.revues.org/4646

Dubois, Jacques. La institución de la literatura. Medellín: Universidad de Antioquia, [1979] 2014.

Ducas, Sylvie, “Prix littéraires en France : consécration ou désacralisation de l'auteur ?”, COnTEXTES, no. 7, junio (2010). Disponible en: http://contextes.revues.org/4656

Fernández Bravo, Álvaro, "Introducción: elementos para una teoría del valor literario", Boletín del Centro de Estudios de Teoría y Crítica Literaria, $\mathrm{n}^{\circ}$. 15, noviembre (2010(, pp. 1-17.

García, Rodrigo. Sociedad de Mejoras Públicas de Medellín. Cien años haciendo ciudad 1899-1999. Medellín: Comfenalco, Subdirección de Educación, Cultura y Bibliotecas, 1997.

Latorre Mendoza, Luis. Historia e historias de Medellín. Medellín: Ediciones Tomás Carrasquilla, Secretaría de Educación y Cultura de Antioquia, [1934] 1972.

Osuna, Rafael. Tiempo, materia y texto. Una reflexión sobre las revistas literarias. Kassel: Edition Reichenberger, 1998.

-Las Revistas Literarias. Un estudio introductorio. Cádiz: Servicio de Publicaciones de la Universidad de Cádiz, 2004.

Scullard, Howard Hayes. Festivals and ceremonies of the Roman Republic. New York: Cornell University Press, 1981.

Tamayo Ortiz, Dora Helena y Hernán Botero Restrepo (comp.). Inicios de una literatura regional. La narrativa antioqueña de la segunda mitad del siglo XIX (1855-1899). Medellín: Universidad de Antioquia, 2005. 